\title{
Glucocorticoid Receptor Gene-Based SNP Analysis in Patients with Recurrent Major Depression
}

\author{
Dirk van West ${ }^{1,2,3}$, Filip Van Den Eede ${ }^{1,2}$, Jurgen Del-Favero', Daniel Souery4, Karl-Fredrik Norrback ${ }^{5}$, \\ Cornelia Van Duijn ${ }^{1,6}$, Sam Sluijs', Rolf Adolfsson ${ }^{5}$, Julien Mendlewicz ${ }^{4}$, Dirk Deboutte ${ }^{2,3}$, \\ Christine Van Broeckhoven' and Stephan Claes*, 1,7 \\ 'Department of Molecular Genetics, Flanders Interuniversity Institute for Biotechnology (VIB), University of Antwerp, Antwerp, Belgium; \\ ${ }^{2}$ Collaborative Antwerp Psychiatric Research Institute (CAPRI), University of Antwerp, Antwerp, Belgium; ${ }^{3}$ University Centre of Child and \\ Adolescent Psychiatry Antwerp (UCKJA), Antwerp, Belgium; ${ }^{4}$ Department of Psychiatry, Erasme Hospital, University of Brussels (ULB), Brussels, \\ Belgium; ${ }^{5}$ Department of Clinical Sciences, Psychiatry, Umeå University, Umeå, Sweden; ${ }^{6}$ Department of Epidemiology \& Biostatistics, Erasmus \\ Medical Center, Rotterdam, The Netherlands; ${ }^{7}$ Department of Psychiatry, University of Leuver, Leuver, Belgium
}

\begin{abstract}
Dysregulation of the hypothalamic-pituitary-adrenal axis, one of the stress-response systems, is one of the key neurobiological features of major depression (MDD). Data supporting the notion that glucocorticoid-mediated feedback inhibition is impaired in MDD come from a multitude of studies demonstrating nonsuppression of cortisol secretion following administration of the synthetic glucocorticoid dexamethasone. We examined whether genetic variations in the glucocorticoid receptor gene (Nuclear Receptor Subfamily 3, Group C, Member I; NR3Cl) could be associated with increased susceptibility for MDD using a whole gene-based association analysis of single nucleotide polymorphisms (SNPs). Four SNPs were identified in NR3CI and genotyped in two well-diagnosed samples of patients with MDD ascertained in Belgium and northern Sweden, and matched control samples. In total, 3 I 4 MDD patients and 354 control individuals were included in the study. In the Belgian sample, we observed significant allele $(p=0.02)$ and genotype $(p=0.02)$ association with an SNP in the promoter region (NR3Cl-I); in the Swedish sample, we observed significant allele $(p=0.02)$ and genotype $(p=0.02)$ association with the R23K SNP. The haplotype association studies showed modest evidence for an involvement of the $5^{\prime}$ region of the $\mathrm{NR3Cl}$ gene in the genetic vulnerability for MDD. This study suggests that polymorphisms in the $5^{\prime}$ region of the $\mathrm{NR} 3 \mathrm{Cl}$ gene may play a role in the genetic vulnerability for MDD.

Neuropsychopharmacology (2006) 31, 620-627. doi:I0.1038/sj.npp. I 300898; published online 28 September 2005
\end{abstract}

Keywords: genetic; glucocorticoid receptor; major depression; HPA axis; stress; polymorphism

\section{INTRODUCTION}

Hyperactivity of the hypothalamic-pituitary-adrenal (HPA) axis in patients with major depression (MDD) is one of the most consistent findings in biological psychiatry. Specifically, patients with MDD were shown to exhibit increased concentrations of cortisol in plasma, urine, and cerebrospinal fluid (CSF); an exaggerated cortisol response to adrenocorticotrophic hormone (ACTH); and an enlargement of both the pituitary and the adrenal glands (Gold et al, 1988; Holsboer and Barden, 1996; Holsboer, 2000; Nemeroff, 1996; Owens and Nemeroff, 1993; Plotsky et al,

\footnotetext{
* Correspondence: Professor Dr S Claes, Department of Molecular Genetics VIB8, Psychiatry Research Group, University of Antwerp, Universiteitsplein I, B-2610 Antwerpen, Belgium, Tel: + 32382023 2I, Fax: +32 382025 4I, E-mail: stephan.claes@ua.ac.be Received 21 March 2005; revised 3 August 2005; accepted II August 2005

Online publication: 18 August 2005 at http://www.acnp.org/citations/ Npp08 | 805050 | 77/default.pdf
}

1998). Moreover, depressed patients exhibit increased concentrations of CRH in the CSF, increased CRH messenger RNA (mRNA) and protein in the paraventricular nucleus (PVN) of the hypothalamus, and a blunted ACTH response to a CRH challenge (Gold et al, 1988; Nemeroff, 1996). A major hypothesis that has been proposed for the pathogenesis of MDD is the corticosteroid receptor hypothesis, which focuses on the impaired corticosteroid receptor signaling as a primary factor in the pathogenesis, leading to a reduced negative feedback of cortisol, an increased production of CRF, and hypercortisolism (Holsboer, 2000).

Corticosteroid signaling by glucocorticoids is mediated through two distinct intracellular receptor subtypes referred as the mineralocorticoid receptor (MR) and the glucocorticoid receptor (GR) (Reul and de Kloet, 1985). The MR has a high affinity for endogenous corticosteroids and is believed to play a role in the regulation of circadian fluctuations in these hormones. In contrast to the MR, the GR has a high affinity for dexamethasone and a lower affinity for 
endogenous corticosteroids. The GR is believed to be more important in the regulation of the response to stress, when endogenous levels of glucocorticoids are high. Spencer et al (1998) and de Kloet et al (1998) have clarified that GR activation is necessary for the HPA feedback regulation when levels of glucocorticoids are high (response to stress, circadian peak), but that the MR also plays an important role by modulating GR-dependent regulation. Because patients with MDD exhibit impaired HPA negative feedback in the context of elevated circulating levels of cortisol and because altered HPA axis responsiveness has been characterized with dexamethasone, which selectively binds GR in vivo, studies investigating corticosteroid receptors in MDD have logically focused on the expression and function of GR.

Three major possibilities have been considered regarding the mechanism(s) of GR resistance in MDD. These include (1) GR downregulation secondary to persistent hypercortisolism, (2) a primary alteration in the genetic structure of the GR, and (3) a decrease in GR function secondary to alterations in ligand-independent pathways that regulate the GR (Bamberger et al, 1996; Boyle et al, 2005; Juruena et al, 2003; Pariante and Miller, 2001).

The human gene coding for the GR is most frequently referred to as Nuclear Receptor Subfamily 3, Group C, Member 1 (NR3C1) and is located on chromosome 5q31q32. The gene comprises nine exons (Nobukuni et al, 1995). Exon 1 and part of exon 2 contain the $5^{\prime}$ UTR, exons 2-9 the coding sequences, and part of exon 9 the $3^{\prime}$ UTR (Nobukuni et al, 1995). Recently, two additional alternative first exons (designated exons $1 \mathrm{~A}$ and $1 \mathrm{~B}$ ) were identified upstream of exon 1 (now exon 1C) (Breslin et al, 2001). At least three promoters regulate the transcriptional activity of NR3C1 (Breslin et al, 2001).

In a recent review, DeRijk et al (2002) described more than 30 genetic variants in NR3C1. Most were confined to one or a few families, and were mutations associated with glucocorticoid resistance and metabolic and cardiovascular function. Some were found to influence basal cortisol levels, the negative HPA axis feedback, and the stress-induced activation of the system. Moutsatsou et al (2000) investigated the coding sequences of the two GR isoforms, GR $\alpha$ and $\mathrm{GR} \beta$, in 15 patients with bipolar affective disorder and 12 normal control individuals; they did not find NR3C1 mutations in the coding sequences. However, this study was not designed to look at association at the haplotype level, and therefore a possible role of SNPs outside the coding regions was not assessed. Feng et al (2000) found no evidence for association between five SNPs in the region coding for the $\mathrm{N}$-terminal domain of the protein $\mathrm{NR} 3 \mathrm{Cl}$ and puerperal bipolar or depressive psychosis or schizophrenia. Wüst et al (2004) investigated the impact of three GR gene polymorphisms (BclI RFLP, N363S, ER22/23EK) on cortisol and ACTH responses to psychosocial stress in normal healthy males; they found that $363 S$ carriers were associated with markedly larger cortisol responses, whereas the mean response in BclI G homozygotes was attenuated. A thorough haplotype-based association analysis of the role of NR3C1 has not yet been reported, to our best knowledge.

This paper is a part of a systematic evaluation of the possible role of a number of HPA axis-related genes in the genetic vulnerability for MDD. Previously, our group published reports on the genes encoding for the CRF receptor 2, the CRF binding protein, and the AVP receptor 1B (Claes et al, 2003; van West et al, 2004; Villafuerte et al, 2002).

The aim of this study was therefore to examine whether genetic variations in NR3C1 might contribute to the liability to develop MDD. Here, we identified SNPs in NR3C1 and subsequently analyzed them in a genetic association study in two geographically different samples of patients with MDD and healthy control individuals.

\section{MATERIALS AND METHODS}

\section{Patient-Control Samples}

Belgian sample. The Belgian patient sample consisted of 180 unrelated MDD patients. The gender ratio was $132 \mathrm{~F} / 48$ $\mathrm{M}$, and mean age at inclusion was 53.1 years \pm 13.5 . Patients were diagnosed by a semistructured interview (Mini International Neuropsychiatric Interview, MINI) (Sheehan et al, 1998) by a trained psychiatrist and diagnoses were made according to the Research Diagnostic Criteria (RDC). All patients had at least two well-defined episodes of MDD as defined by DSM-IV (American Psychiatric Association, 1994). Family data were assessed using the Family History RDC instrument (Andreasen et al, 1977). When available, family data were also collected from relatives. All individuals were ascertained at the Department of Psychiatry of the Erasme Hospital in Brussels, Belgium.

In the Belgian control group $(n=173)$, individuals with a positive personal history of psychiatric disorder were excluded using the Schedule for Affective Disorders and Schizophrenia-Lifetime Version (SADS-LA40). The gender ratio was $109 \mathrm{~F} / 64 \mathrm{M}$, and mean age was 51.6 years \pm 13.0 . Family history of psychiatric disorders was assessed using the Family History RDC instrument (Andreasen et al, 1977), and individuals with first-degree relatives showing mood disorders were excluded. There were no statistically significant differences between patient and control individuals for age and ethnicity; a borderline significant difference between patient and control individuals was found for gender.

Swedish sample. The Swedish patient sample consisted of 134 unrelated MDD patients. The gender ratio was $87 \mathrm{~F} / 47$ $M$, and the mean age at inclusion was 68.6 years \pm 15.0 . Patients were diagnosed using a semistructured interview (MINI) (Sheehan et al, 1998) by a trained psychiatrist and diagnoses were made according to the RDC. All patients had at least two well-defined episodes of MDD as defined by DSM-IV (American Psychiatric Association, 1994).

The Swedish control individuals $(n=181)$ were selected from a random population-based study. Individuals with a lifetime history of psychotic disorders according to a clinical interview were excluded. This means that no individuals with a lifetime history of schizophrenia or of bipolar disorder were included in the Swedish control population, but it is not excluded that a small minority of these individuals has suffered from MDD without psychotic features. 
Individuals with a lifetime history of psychotic disorders according to a clinical interview were excluded. The gender ratio was $117 \mathrm{~F} / 64 \mathrm{M}$, and the mean age 71.6 years \pm 11.6 .

There were no statistically significant differences between patient and control individuals for age, gender, and ethnicity. All originated from the same geographical region in the north of Sweden (County of Väserbotten), and were ascertained at the Department of Psychiatry of the University of Umeå, Sweden.

All patients and control individuals had grandparents of Swedish or Belgian nationality. respectively. The Medical Ethical Committees of the Universities of Umeå, Brussels, and Antwerp approved the research project, and patients were included after having signed an informed consent.

\section{SNP Detection by Sequencing}

Genomic DNAs extracted from whole blood of 24 unrelated Belgian affective disorder patients were used to detect SNPs in NR3C1. This allows to detect all SNPs with a frequency of the minor allele of $2 \%$ or more.

The use of DNA of patients is expected to increase the likelihood that rare SNPs, enriched in patients, are detected. Approximately $10 \mathrm{ng}$ of genomic DNA and $10 \mathrm{pmol}$ of each primer were used in a standard PCR reaction. More information on the sequencing primers is shown in Table 1 (GenBank acc. no. NM_000176).

Direct sequencing of PCR products was performed using the Big Dye terminator cycle sequencing kit v3.0 (Applied Biosystems, $\mathrm{PE}$ ) according to the manufacturer's protocol. Sequencing reactions were run on an ABI 3700 automated sequencer. Data were collected and analyzed using the ABI DNA sequencing software version 3.6. SNPs were identified using the SeqManII software version 4 (DNASTAR Inc.). PCR products where purified using Exo-SAP-It (Amersham Bioscience) as described by the manufacturer. Purified fragments were bidirectional sequenced.

\section{SNP Analysis by Pyrosequencing}

Biotinylated PCR products were immobilized onto streptavidin-coated paramagnetic beads (Dynal AS, Oslo, Norway). Biotinylated ssDNA was obtained by incubating the immobilized PCR product in $50 \mu \mathrm{l}$ of $0.5 \mathrm{M} \mathrm{NaOH}$ for $5 \mathrm{~min}$ followed by two sequential washes in $100 \mu \mathrm{l}$ of $10 \mathrm{mM}$ Tris-acetate $\mathrm{pH}$ 7.6. Primer annealing was obtained by incubation at $80^{\circ} \mathrm{C}$ for $2 \mathrm{~min}$ and then at room temperature for $5 \mathrm{~min}$. Pyrosequencing was performed on the PSQ96 pyrosequencer (Pyrosequencing AB, Uppsala, Sweden).

\section{Statistical Analysis}

The GENEPOP program (Raymond and Rousset, 1995) was used to compare overall allele and genotype distributions for each SNP in MDD patients and controls and to test Hardy-Weinberg equilibrium. GENEPOP estimates exact $p$ values using the Markov chain method; for all four analyses, the dememorization number used was 1000, with 1000 batches and 10000 iterations per batch. The corresponding exact $p$-values were not corrected for multiple testing. To estimate SNP haplotype frequencies in patients and control individuals, the expectation maximization (EM) algorithm
Table I Primers Used for SNP Detection

\begin{tabular}{|c|c|c|c|}
\hline Location & Orientation & Primer & $T_{m}$ \\
\hline \multirow[t]{2}{*}{ Prom A } & $\mathrm{F}$ & CTCCTITCTCAGGACGGACCAC & 64 \\
\hline & $\mathrm{R}$ & CGGCGCATACGTACTITGG & 63 \\
\hline \multirow[t]{2}{*}{ Prom B } & $\mathrm{F}$ & TGGGTTCTGCTTTGCAACTTCTC & 65 \\
\hline & $\mathrm{R}$ & CACACACGCGCTCCCACT & 65 \\
\hline \multirow[t]{2}{*}{ Prom C } & $\mathrm{F}$ & CTITATTAGCCTCGGGGAGTGG & 64 \\
\hline & $\mathrm{R}$ & GCGAGGTTAAAAGAGAAGTACGTCCA & 65 \\
\hline \multirow[t]{2}{*}{ Exon I } & $\mathrm{F}$ & GGATTCTGTGGGTGGAAGGAGAC & 65 \\
\hline & $\mathrm{R}$ & CCTGCGGAGGGAGAGGAAG & 65 \\
\hline \multirow[t]{2}{*}{ Exon $2 \mathrm{a}$} & $\mathrm{F}$ & AAAGGTTCATTTAACAAGCTGCCTCT & 64 \\
\hline & $\mathrm{R}$ & CAGCAGTGGATGCTGAACTCTTG & 65 \\
\hline \multirow[t]{2}{*}{ Exon $2 b$} & $\mathrm{~F}$ & AGGGCCAAATCAGCCTTCCT & 65 \\
\hline & $\mathrm{R}$ & CCCCAGGGGTGCAGAGTTC & 65 \\
\hline \multirow[t]{2}{*}{ Exon 2c } & $\mathrm{F}$ & GGATAATGGAGATCTGGTITTGTCA & 62 \\
\hline & $\mathrm{R}$ & TТТCСТACTTTCAAAAGGCCACTTA & 62 \\
\hline \multirow[t]{2}{*}{ Exon 3} & $\mathrm{~F}$ & CTGCTAGCACTTGAAGCCAGAG & 62 \\
\hline & $\mathrm{R}$ & TGTITAAATACTTTCCTGCCCATT & 61 \\
\hline \multirow[t]{2}{*}{ Exon 4} & $\mathrm{~F}$ & CAGTTTGTTGAACAGAAAAAGGGAGA & 64 \\
\hline & $\mathrm{R}$ & CAGTGTGTGTAAGAAGAACTGGTGGA & 64 \\
\hline \multirow[t]{2}{*}{ Exon 5} & $\mathrm{~F}$ & ТТСТССТТТССАТGTСAСТТАТСA & 62 \\
\hline & $\mathrm{R}$ & GTCCCCAGAACTAAGAGAAACAAGA & 61 \\
\hline \multirow[t]{2}{*}{ Exon 6} & $\mathrm{~F}$ & CAAGAGGGTTTGTGAGTCTTAAAGTG & 62 \\
\hline & $\mathrm{R}$ & CATGTCCTGGGACAGTAATAATGC & 62 \\
\hline \multirow[t]{2}{*}{ Exon7 } & $\mathrm{F}$ & TTGCAGTAGTTGTTITCTTTATGA & 57 \\
\hline & $\mathrm{R}$ & CTATGCAGCTCATATACСTCTCTGT & 58 \\
\hline \multirow[t]{2}{*}{ Exon 8} & $\mathrm{~F}$ & CCTITTAGTTCCTAAGGACGGTCTGA & 64 \\
\hline & $\mathrm{R}$ & CTCAAGCTATCACCAACATCCACA & 63 \\
\hline \multirow[t]{2}{*}{ Exon 9a } & $\mathrm{F}$ & TITTCATCTGGGGAATTCCAGT & 63 \\
\hline & $\mathrm{R}$ & TCAGGTTTCCATGCATAAATCAAA & 63 \\
\hline \multirow[t]{2}{*}{ Exon 9b } & $\mathrm{F}$ & TGGATGAAATTITCTAGACTTTCTG & 58 \\
\hline & $\mathrm{R}$ & TCAGTAGCTGAGCTTTCCTGTACCA & 64 \\
\hline \multirow[t]{2}{*}{ Exon $9 c$} & $\mathrm{~F}$ & AATATGGCAAAAATGGCTAGACACC & 63 \\
\hline & $\mathrm{R}$ & TGTAAAGCTGCAGTAGCCCTTCC & 64 \\
\hline \multirow[t]{2}{*}{ Exon 9d } & $\mathrm{F}$ & CAGGAGACAGGAAGGTGGTGCT & 65 \\
\hline & $\mathrm{R}$ & GCCAAGATTGTTGGGATGAAAATC & 65 \\
\hline \multirow[t]{2}{*}{ Exon $9 e$} & $\mathrm{~F}$ & TTGGTGCCTAAGAAAACTGCTTGA & 64 \\
\hline & $\mathrm{R}$ & CCCCAAAAGTGTTATGTCCTAAGTGC & 65 \\
\hline \multirow[t]{2}{*}{ Exon $9 f$} & $\mathrm{~F}$ & TAAACCCTITGGGTGGAGTTTCG & 65 \\
\hline & $\mathrm{R}$ & ACAGTCTGACATTTCACTGCGTAGGT & 64 \\
\hline
\end{tabular}

F: forward; R: reverse; $T_{m}$ : melting temperature.

provided by the STATA statistical package was applied (http://www-gene.cimr.cam.ac.uk/clayton/software/stata). A two-tailed significance level of $5 \%$ was used for each test.

\section{RESULTS}

\section{SNP Detection and Genotyping}

Validation of publicly available SNPS. Existing databases were consulted for known SNPs within NR3C1. The NCBI SNP database (http://www.ncbi.nlm.nih.gov/SNP/) and the HGBase variation database (http://www.hgbase.de/) 
contained 14 SNPs. Three of the 14 database SNPs were polymorphic (HGBASE reference 6633, 6664, and 6656) by PCR amplification in 24 patients. These SNPs are R23K, N363S, and N766N, respectively.

Detection of novel SNPS. Subsequently, primer pairs were designed to amplify all exons and flanking intron sequences. PCR products were analyzed for sequence variations using sequencing. This resulted in the one additional SNP in the promoter sequence, which we labeled NR3C1-1. The location and exact nomenclature of the four NR3C1 SNPs are shown in Table 2 and schematically represented in Figure 1. Two SNPs in exon 2 of NR3C1 predict amino-acid substitutions.

The four NR3C1 SNPs were subsequently genotyped by pyrosequencing in two patient-control samples ascertained in northern Sweden and Belgium (Table 3). In both control populations, the allele and genotype distributions of all four SNPs were in Hardy-Weinberg equilibrium $(p>0.05)$. Minor allele frequencies of the four SNPs varied from 2 to $24 \%$ in northern Swedish, and from 3 to $14 \%$ in Belgian control individuals (Table 3).

\section{SNP Association Analysis}

In the Belgian patient-control sample, a significant difference in allele distribution between patients and controls was observed for NR3C1-1 $(p=0.02)$ (Table 3), with the minor allele $\mathrm{C}$ more frequently present in controls. For the Swedish patient-control sample, a significant difference in allele distribution between patients and controls was observed for $R 23 K(p=0.02)$ (Table 3), with the minor allele A more frequently present in patients. In the genotype distributions, this is reflected by a significant increase of CT heterozygotes of NR3C1-1 in the Belgian controls $(p=0.02)$ and of AG heterozygotes of $R 23 \mathrm{~K}$ $(p=0.02)$ in the Swedish patients (Table 3).

\section{SNP Haplotype Analysis}

We estimated the SNP haplotype frequencies in patients and control individuals using the EM algorithm embedded in the program STATA. In the Belgian patient-control sample, six haplotypes represented $>99 \%$ of the total haplotype diversity present in patients and control subjects. The overall distribution of the six haplotypes was significantly different between patients and controls $(p=0.04)$. In the Swedish patient-control sample, five haplotypes represented $>98 \%$ of the total haplotype diversity present in patients and control subjects. Also here, the overall distribution of these five haplotypes was significantly different between patients and controls $(p=0.03)$.

When looking at the sliding window of respectively two, three, and four SNPs (Table 4), in the Swedish sample all haplotypes in which SNP NR3C1-1 or R23K are included show a significant difference in haplotype distribution between patients and controls. The main haplotype driving the significant finding is the CAAT haplotype, which was found in 5\% of MDD patients and in $1 \%$ of control individuals. Only the two-SNP window containing N363S$N 766 N$ yields no evidence for association. In the Belgian sample, all windows including SNP NR3C1-1 show a significant difference in haplotype distribution between MDD and control individuals. Here, the positive association is most probably due to haplotype CGAT with a frequency of $7 \%$ in MDD patients and $11 \%$ in controls.

Table 2 Location, Nomenclature, and Primers of Four SNPs in NR3CI

\begin{tabular}{|c|c|c|c|c|c|c|}
\hline Name & $\begin{array}{l}\text { Genomic } \\
\text { location }\end{array}$ & $\begin{array}{l}\text { Nucleotide } \\
\text { change }\end{array}$ & $\begin{array}{l}\text { Amino-acid } \\
\text { change }\end{array}$ & $\begin{array}{l}\text { dbSNP } \\
\text { reference }\end{array}$ & PCR primers & SNP primers \\
\hline $\mathrm{NR3CI}-\mathrm{I}$ & Promoter & g. -321 IT $>C$ & - & rs 10482605 & F-GAGCTCCCGAGTGGGTCT & CCAACACCCAGGAAAA \\
\hline \multirow[t]{2}{*}{ R23K } & Exon 2 & c. $68 \mathrm{G}>\mathrm{A}$ & Arg23Lys & rs6190 & F-CAAGCTGCCTCTTACTAATCG & CCCAGGTCATTTCCCATCAC \\
\hline & & & & & R-CCCAGGTCATTTCCCATCAC & \\
\hline \multirow[t]{2}{*}{ N766N } & Exon 9alfa & c.2298T > C & - & rs6196 & F-GGGAATTCCAGTGAGATTGG & AGAAGTITITGATA \\
\hline & & & & & R-CAACTGCTTCTGTTGCCAAG & \\
\hline
\end{tabular}

F: forward; R: reverse.

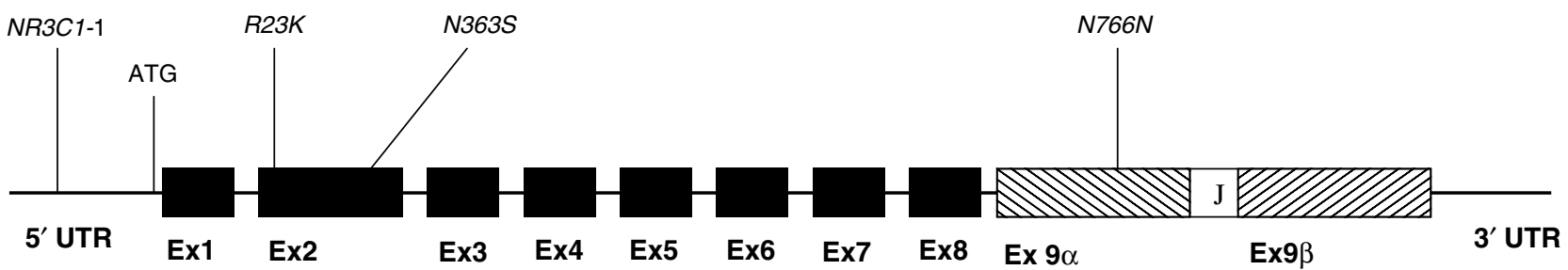

Figure I Schematic representation of the genomic localization of four SNPs of NR3CI (Table I). 
Table 3 Genotypic and Allelic Distribution (\%) of NR3CI SNPs in MDD Patients and Control Individuals (CO)

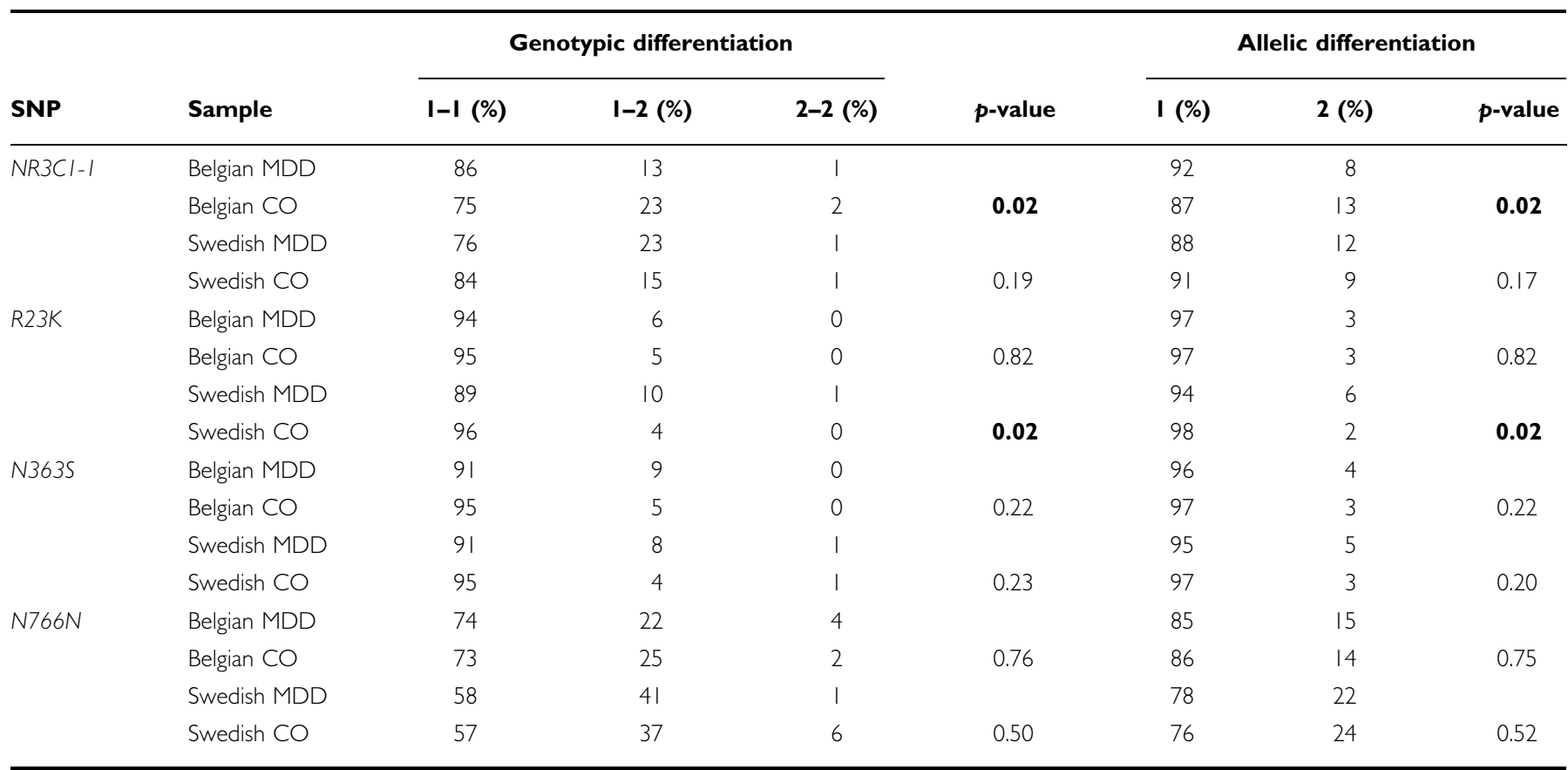

GENEPOP estimates exact $p$-values using the Markov chain method. The SNP alleles were coded as follows: NR3CI-I: I = T, 2=C; R23K: I = G, 2=A; N363S: $\mathrm{I}=\mathrm{A}, 2=\mathrm{G} ; \mathrm{N} 766 \mathrm{~N}: \mathrm{I}=\mathrm{T}, 2=\mathrm{C}$.

$P$-values $<0.05$ are indicated in bold.

In all samples, strong LD was observed between NR3C1-1 and $R 23 \mathrm{~K}$. However, there was no significant evidence for LD for all other SNP combinations. This could be due to the low frequency of the minor allele of SNPs R23K and N363S.

In principle, it is possible to translate the positive haplotype association findings into odds ratios. For example, the CA haplotype for SNPs NR3C1-1/R23K, which was associated with the Swedish population (cf Table 4), results in an increased risk for MDD with an odds ratio of 4.58 (CI 1.48-14.21). However, given the uncertainty as to which haplotype specifically increases the risk, it did not seem particularly useful to calculate odds ratios for all positive association findings.

\section{DISCUSSION}

This study offers a systematic detection of SNPs in NR3C1 and subsequent association analysis in two clinically welldefined samples of MDD patients and matched control individuals ascertained in northern Sweden and Belgium. We compared allele and genotype frequencies of four SNPs in the Belgian and Swedish MDD patients and control individuals. In the Belgian sample, one SNP in the promoter region showed a significant association with MDD; in the Swedish sample, one SNP in exon 2 showed a significant association with the disorder.

By evaluating haplotypes rather than single loci, the loss of information attributable to biallelic rather than multiallelic loci can be compensated, potentially resulting in increased informativity in the association analysis. The haplotype analysis shows that in both populations, haplotypes containing SNPs NR3C1-1 and/or R23K show evidence for association. SNPs NR3C1-1, R23K, and N363S are located relatively close together, whereas SNP N766N is separated by about $100 \mathrm{~kb}$ from SNP N363S. According to HAPMAP (www.hapmap.org), the GR gene contains several haplotype blocks. This is the case using the four-gamete approach; other approaches, including the conservative approach of Gabriel, suggest that HAPMAP data indicate a large region of LD in this area. This is also generally borne out by newer Perlegen data. The data are compatible with an associated haplotype spanning part of the promoter region and of the $5^{\prime}$ region of the gene.

SNP N363S causes an asparagine-to-serine change in codon 363 of the NR3C1 protein. This polymorphism has been related to an altered sensitivity to glucocorticoids (Huizenga et al, 1998). In a group of 216 elderly persons, they identified 13 heterozygotes for the asn363-to-ser polymorphism by PCR/SSCP analysis. Thus, they found the polymorphism in $6.0 \%$ of the studied population. Huizenga et al (1998) concluded that individuals carrying this polymorphism were clinically healthy, but had a higher sensitivity to exogenously administered glucocorticoids, with respect to both cortisol suppression and insulin response. Huizenga et al (1998) further speculated that lifelong exposure to the mutated allele may be accompanied by an increased body mass index and a lowered bone mineral density in the lumbar spine with no effect on blood pressure. However, it is improbable that in our study the codon 363 SNP drives the association at the haplotype level, since it does not show evidence for association in the single SNP association approach. SNP R23K leads to an Arg23Lys mutation, which has been associated with (relative) resistance to glucocorticosteroids (van Rossum et al, 2002). This polymorphism shows evidence for association 
Table 4 Haplotype Analyses Using a Sliding Window of Two, Three, and Four SNPs

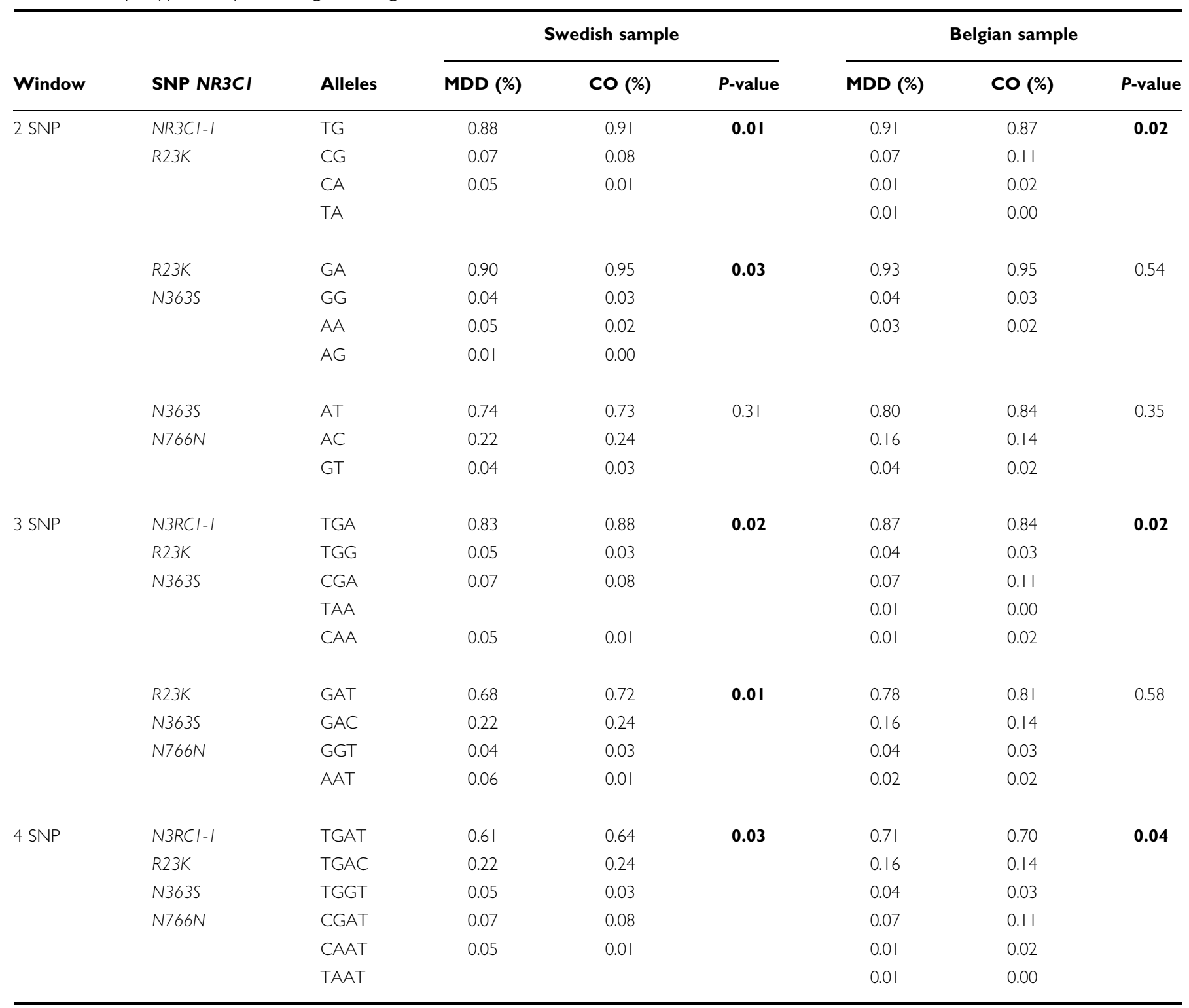

The expectation maximization (EM) algorithm provided by STATA statistical package was applied.

$P$-values $<0.05$ are indicated in bold.

in the Swedish sample, but not in the Belgian one. It is impossible to conclude from our study whether this SNP is responsible for the association seen at the haplotype level. However, Russcher et al (2005) postulated that a higher expression of the less transcriptionally active GR-A isoforms, and thus a lower expression of the more transcriptionally active GR-B isoforms mainly cause a decrease in glucocorticoid sensitivity. This shift in GR-A/GR-B expression ratio is evoked by the ER22/23EK polymorphism, possibly by changing the secondary structure of the mRNA of the GR, causing more translation initiation from the first AUG start site.

A first point of concern might be that the association at the haplotype level is mainly driven by rare haplotypes, with frequencies around 5\%, and that the estimation of rare haplotypes with EM algorithms might be less reliable. However, in a recent study, Adkins (2004) showed that the estimation of haplotypes with a frequency of $1 \%$ or more is highly reliable using an EM approach. Furthermore, the accuracy of the haplotype estimation method in this study can be appreciated by comparing the estimated number of homozygotes for the major haplotype with the actually counted number of homozygotes. For the Belgian population, the frequency of the major haplotype was $71 \%$ in both patients and control individuals, implying a homozygosity frequency of $50 \%$ in both groups. Actual counting showed frequencies of 53 and $52 \%$, respectively, in patients and control individuals. In the Swedish population, the major haplotype had an estimated frequency of $61 \%$ in patients and $63 \%$ in control individuals. This implies that the homozygosity frequency for this haplotype was 37 and $40 \%$, respectively, assuming that the haplotype distribution in the population is in Hardy-Weinberg equilibrium. Actual counting of homozygotes showed a frequency of $38 \%$ in 
patients and $40 \%$ in controls. These data support the accuracy of the haplotype estimations.

A second point of concern is the risk of false positive findings due to multiple comparisons. After Bonferroni correction for multiple testing, the significance of the SNP association findings will be reduced. However, in the sliding window haplotype association analysis, eight out of 12 distribution comparisons showed $p<0.05$, specifically these analyses containing one or more SNPs located at the $5^{\prime}$ end of the gene. This is unlikely to be caused by chance. Furthermore, the six haplotype distribution comparisons in each sample are all strongly dependent upon each other, implying that a correction for six independent tests would be much too conservative. Therefore, the significance of the haplotype association findings would survive appropriate correction for multiple testing.

To summarize, the results of this study imply that a genetic variation in the promoter region or the $5^{\prime}$ end of the $N R 3 C 1$ gene plays a role in the vulnerability for MDD in the general population. However, the results obtained in both populations are quite different, and no corrections were made for multiple testing; therefore, these results should be interpreted cautiously. Other genetic factors may also lead to a dysfunction of the GR. Recently, Binder et al (2004) found that an intronic polymorphism in FKBP5, a GRregulating cochaperone of hsp-90, was highly associated with increased response to antidepressant treatment and postulated a central role of genes regulating the HPA axis in the causality of depression and the mechanism of action of antidepressant drugs. Clearly, the function of the HPA axis and the clinical appearance of MDD are complex phenotypes, influenced by many genes and environmental conditions, most single genes probably accounting for only a small proportion of phenotype variability.

A next logical step would be to sequence the whole associated $5^{\prime}$ region in all patients and controls, in order to reveal all the genetic variation present there. Subsequently, functional studies would be needed to determine the impact of each SNP on the expression of the GR gene, and to identify the SNP that underlies the association.

\section{ACKNOWLEDGEMENTS}

We acknowledge the VIB Genetic Service Facility (http:// www.vibgeneticservicefacility.be/) for the genetic analyses. The project was in part funded by the Special Research Fund of the University of Antwerp (UA), the Fund for Scientific Research Flanders (FWO), the Fond National de la Recherche Scientific (FNRS), and the Interuniversity Attraction Poles program P5/19 of the Federal Science Policy Office, Belgium. The Torsten and Ragnar Söderbergs Foundation, Medical Research Council K2001-21X-1041209A, and the County Council of Västerbotten, Sweden supported the ascertainment of the Swedish patient-control sample.

\section{REFERENCES}

Adkins RM (2004). Comparison of the accuracy of methods of computational haplotype inference using a large empirical dataset. BMC Genet 5: 22-29.
American Psychiatric Association (1994). Diagnostic and Statistical Manual for Mental Disorders. American Psychiatric Press: Washington, DC.

Andreasen NC, Endicott J, Spitzer RL, Winokur G (1977). The family history method using diagnostic criteria. Reliability and validity. Arch Gen Psychiatry 34: 1229-1235.

Binder EB, Salyakine D, Lichtner P, Wochnik GM, Ising M, Pütz B et al (2004). Polymorphisms in FKBP5 are associated with increased recurrence of depressive episodes and rapid response to antidepressant treatment. Nat Genet 36: 1319-1325.

Bamberger CM, Schulte HM, Chrousos GP (1996). Molecular determinants of glucocorticoid receptor function and tissue sensitivity to glucocorticoids. Endocr Rev 17: 245-261.

Boyle MP, Brewer JA, Funatsu M, Wozniak DF, Tsien JZ, Izumi Y et al (2005). Acquired deficit of forebrain glucocorticoid receptor produces depression-like changes in adrenal axis regulation and behavior. Proc Natl Acad Sci USA 102: 473-478.

Breslin MB, Geng CD, Vedeckis WV (2001). Multiple promoters exist in the human GR gene, one of which is activated by glucocorticoids. Mol Endocrinol 15: 1381-1395.

Claes S, Villafuerte S, Forsgren T, Sluijs S, Del-Favero J, Adolfsson $\mathrm{R}$ et al (2003). The corticotropin-releasing hormone binding protein is associated with major depression in a population from Northern Sweden. Biol Psychiatry 54: 867-872.

De Kloet ER, Vreugdenhil E, Oitzl MS, Joels M (1998). Brain corticosteroid receptor balance in health and disease. Endocr Rev 19: 269-301.

DeRijk RH, Schaaf M, de Kloet ER (2002). Glucocorticoid receptor variants: clinical implications. J Steroid Biochem Mol Biol 81: $103-122$.

Feng J, Zheng J, Bennett WP, Heston LL, Jones IR, Craddock N et al (2000). Five missense variants in the amino-terminal domain of the glucocorticoid receptor: no association with puerperal psychosis or schizophrenia. Am J Med Genet 12: 412-417.

Gold PW, Goodwin FK, Chrousos GP (1988). Clinical and biochemical manifestations of depression. Relation to the neurobiology of stress. $N$ Engl J Med 319: 413-420.

Holsboer F, Barden N (1996). Antidepressants and hypothalamicpituitary-adrenocortical regulation. Endocr Rev 17: 187-205.

Holsboer F (2000). The corticosteroid hypothesis of depression. Neuropsychopharmacology 23: 477-501.

Huizenga N, Koper J, de Lange P, Pols H, Stolk R, Burger H et al (1998). A polymorphism in the glucocorticoid receptor gene may be associated with an increased sensitivity to glucocorticoids in vivo. J Clin Endocr Metab 83: 144-151.

Juruena MF, Cleare AJ, Bauer ME, Pariante CM (2003). Molecular mechanisms of glucocorticoid receptor sensitivity and relevance to affective disorders. Acta Neuropsychiatrica 15: 354-367.

Moutsatsou P, Tsolakidou A, Trikkas G, Troungos C, Sekeris CE (2000). Glucocorticoid receptor alpha and beta isoforms are not mutated in bipolar affective disorder. Mol Psychiatry 5: 196-202.

Nemeroff CB (1996). The corticotropin-releasing factor (CRF) hypothesis of depression: new findings and new directions. Mol Psychiatry 1: 336-342.

Nobukuni Y, Smith CL, Hager GL, Detera Wadleigh SD (1995). Characterization of the human glucocorticoid receptor promoter. Biochemistry 34: 8207-8214.

Owens MJ, Nemeroff CB (1993). The role of corticotropin-releasing factor in the pathophysiology of affective and anxiety disorders: laboratory and clinical studies. Ciba Found Symp 172: 296-308.

Pariante CM, Miller AH (2001). Glucocorticoid receptors in major depression: relevance to pathophysiology and treatment. Biol Psychiatry 49: 391-404.

Plotsky PM, Owens MJ, Nemeroff CB (1998). Psychoneuroendocrinology of depression. Hypothalamic-pituitary-adrenal axis. Psychiatr Clin N Am 21: 293-307. 
Raymond M, Rousset F (1995). GENEPOP (version 1.2). A population genetics software for exact tests and ecumenicism. J Hered 86: 248-249.

Reul JM, de Kloet ER (1985). Two receptor systems for corticosterone in rat brain: microdistribution and differential occupation. Endocrinology 117: 2505-2511.

Russcher H, van Rossum E, de Jong F, Brinkmann A, Lamberts S, Koper J (2005). Increased expression of the glucocorticoid receptor: a translation isoforms as a result of the ER22/23EK polymorphism. Mol Endocinol 19: 1687-1696.

Sheehan DV, Lecrubier Y, Sheehan KH, Amorim P, Janavs J, Weiler E (1998). The Mini-International Neuropsychiatric Interview (M.I.N.I.): the development and validation of a structured diagnostic psychiatric interview for DSM-IV and ICD-10. J Clin Psychiatry 59(Suppl): 22-33.

Spencer RL, Kim PJ, Kalman BA, Cole MA (1998). Evidence for mineralocorticoid receptor facilitation of glucocorticoid receptor-dependent regulation of hypothalamic-pituitary-adrenal axis activity. Endocrinology 139: 2718-2726. van Rossum E, Koper J, Huizenga N, Uitterlinden A, Janssen J, Brinkmann A et al (2002). A polymorphism in the glucocorticoid receptor gene, which decreases sensitivity to glucocorticoids in vivo, is associated with low insulin and cholesterol levels. Diabetes 51: 3128-3134.

van West D, Del-Favero J, Aulchenko Y, Oswald P, Souery D, Forsgren T et al (2004). A major SNP haplotype of the arginine vasopressin $1 \mathrm{~b}$ receptor protects against recurrent major depression. Mol Psychiatry 9: 287-292.

Villafuerte S, Del-Favero J, Adolfsson R, Souery D, Massat I, Mendlewicz J et al (2002). Gene-based SNP genetic association study of the corticotropin-releasing hormone receptor-2 (CRHR2) in major depression. Am J Med Genet 114: 222-226.

Wüst S, Van Rossum EF, Frederenko IS, Koper JW, Kumsta R, Hellhammer DW (2004). Common polymorphisms in the glucocorticoid receptor gene are associated with adrenocortical responses to psychosocial stress. J Clin Endocrinol Metab 89: $565-573$. 